\title{
OPEN Transcriptional overlap links DNA hypomethylation with DNA hypermethylation at adjacent promoters in cancer
}

\begin{abstract}
Jean S. Fain ${ }^{1}$, Axelle Loriot ${ }^{1,2}$, Anna Diacofotaki ${ }^{1}$, Aurélie Van Tongelen ${ }^{1} \&$ Charles De Smet $^{1 凶}$
Tumor development involves alterations in DNA methylation patterns, which include both gains (hypermethylation) and losses (hypomethylation) in different genomic regions. The mechanisms underlying these two opposite, yet co-existing, alterations in tumors remain unclear. While studying the human MAGEA6/GABRA3 gene locus, we observed that DNA hypomethylation in tumor cells can lead to the activation of a long transcript (CT-GABRA3) that overlaps downstream promoters (GABRQ and $G A B R A 3)$ and triggers their hypermethylation. Overlapped promoters displayed increases in H3K36me3, a histone mark deposited during transcriptional elongation and known to stimulate de novo DNA methylation. Consistent with such a processive mechanism, increases in $\mathrm{H} 3 \mathrm{~K} 36 \mathrm{me} 3$ and DNA methylation were observed over the entire region covered by the CT-GABRA3 overlapping transcript. Importantly, experimental induction of CT-GABRA3 by depletion of DNMT1 DNA methyltransferase, resulted in a similar pattern of regional DNA hypermethylation. Bioinformatics analyses in lung cancer datasets identified other genomic loci displaying this process of coupled DNA hypo/hypermethylation, and some of these included tumor suppressor genes, e.g. RERG and PTPRO. Together, our work reveals that focal DNA hypomethylation in tumors can indirectly contribute to hypermethylation of nearby promoters through activation of overlapping transcription, and establishes therefore an unsuspected connection between these two opposite epigenetic alterations.
\end{abstract}

Cancer development is driven in part by the accumulation of epigenetic alterations, which render chromatin permissive to changes in gene expression patterns. As a result, tumor cells acquire increased plasticity, thereby facilitating their evolution towards full malignancy ${ }^{1}$. Epigenetic alterations concern in particular DNA methylation, a chemical modification of cytosines in $\mathrm{CpG}$ sequences that is associated with long-term transcriptional repression ${ }^{2}$. DNA methylation changes in tumors include gains (hypermethylation) within normally unmethylated gene promoters, and at the same time losses (hypomethylation) in other genomic sequences ${ }^{3}$. The mechanisms underlying these contrasting changes in DNA methylation patterns in tumors are only partially elucidated, and evidence so far suggest that DNA hypermethylation and hypomethylation result from two independent processes $^{4,5}$.

DNA hypermethylation has a well-established role in tumor progression, as it can lead to irreversible silencing of genes with tumor suppressor functions ${ }^{6}$. DNA hypomethylation on the other hand, appears to promote tumor development by increasing genomic instability, and by inducing ectopic activation of genes with oncogenic functions ${ }^{7}$. Although genes ectopically activated in hypomethylated tumors were reported to display different tissue specificities ${ }^{8}$, most of them were found to belong to the class of so-called "cancer-germline" (CG) genes, as their expression in healthy adults is normally restricted to testicular germ cells ${ }^{9}$. It has indeed been demonstrated that CG genes rely primarily on DNA methylation for repression in non-expressing cells, and that DNA demethylation is a sufficient trigger for their activation in a variety of tumors ${ }^{10-12}$. Evidence has accumulated indicating that some CG genes contribute to tumor progression, notably by encoding proteins that regulate processes of cell proliferation, death resistance, metabolic adaptation, and DNA repair ${ }^{13,14}$.

Recently, we discovered a novel CG transcript (CT-GABRA3) showing DNA hypomethylation-dependent activation in a variety of tumors, including melanoma and lung cancer ${ }^{15,16}$. The CT-GABRA3 transcript is noncoding, extends over a large distance $(530 \mathrm{~kb})$, and overlaps the Gamma-Aminobutyric Acid Type A Receptor

${ }^{1}$ Group of Genetics and Epigenetics, de Duve Institute, Université Catholique de Louvain, Brussels, Belgium. ${ }^{2}$ Group of Computational Biology and Bioinformatics, de Duve Institute, Université Catholique de Louvain, Brussels, Belgium. ${ }^{\boxplus}$ email: charles.desmet@uclouvain.be 
Subunit Alpha3 (GABRA3) gene, starting $250 \mathrm{~kb}$ downstream. Of note, CT-GABRA3 shares a bidirectional promoter with the well described MAGEA6 CG gene, and both transcripts are most often co-expressed. An intriguing observation was that in the melanoma cell lines where we detected hypomethylation and activation of MAGEA6/CT-GABRA3, the promoter of GABRA3 exhibited marked hypermethylation. This suggested that promoter hypomethylation and subsequent transcriptional activation of CT-GABRA3 might trigger DNA hypermethylation of the downstream GABRA3 overlapped promoter. Epigenetic processes involving overlapping transcription have indeed been implicated in the establishment of DNA methylation marks at parentally imprinted sites, and in intragenic promoters during development ${ }^{17,18}$. The underlying mechanism involves deposition of H3K36me3 modification along with the transcriptional machinery, and consequent recruitment of de novo DNA methyltransferases. This mechanism also explains the fact that actively transcribed genes usually show higher CpG methylation within their body, probably as a way to prevent spurious transcription initiation downstream of the transcription start site ${ }^{19-21}$.

In the present study, we set out to validate the relationship between the methylation status of CT-GABRA3 and GABRA3 promoters in tumors. The involvement of transcriptional overlap in this process was investigated. We then used a bioinformatics approach to explore the possibility that this process of coupled hypo/hypermethylation of CpGs occurs in other parts of the genome of cancer cells, and contributes to DNA hypermethylation of tumor suppressor genes.

\section{Results}

MAGEA6/CT-GABRA3 promoter hypomethylation correlates with GABRA3 promoter hypermethylation in melanoma. Recently, our studies focused on the human MAGEA6/GABRA3 gene locus on chromosome $\mathrm{X}^{15,16}$. We demonstrated the existence of a bidirectional promoter (MAGEA6/CT-GABRA3) driving expression of both MAGEA6 and CT-GABRA3 transcripts in testis. CT-GABRA3 starts $\sim 250-\mathrm{kb}$ upstream of another gene (GABRA3), comprises several specific exons in its 5' part, and then overlaps GABRA3, of which it comprises all but exon 1 (Fig. 1A). MAGEA6 and CT-GABRA3 become aberrantly co-activated in a significant proportion of tumors, including melanoma, and our previous studies indicated that this was caused by DNA demethylation of the MAGEA6/CT-GABRA3 promoter $^{15,16}$. Evidence for this was in part provided by bisulfite sequencing results, as shown in Fig. 1B, showing that the MAGEA6/CT-GABRA3 promoter is initially methylated in normal melanocytes, the cell of origin of melanoma. Contrastingly, in melanoma cells where MAGEA6 and CT-GABRA3 are activated, the promoter is completely demethylated, including at critical CpG sites located near the transcription start sites (Fig. 1B). We showed previously that methylated CpGs located near the TSS are most critical for transcriptional repression of CG genes ${ }^{10}$. In the present study, we analyzed the methylation status of the GABRA3 promoter, which normally remains poorly methylated in all tissues (supplementary Fig. S1). Intriguingly, DNA hypermethylation of the GABRA3 promoter was observed specifically in the melanoma cells where the promoter of CT-GABRA3 was hypomethylated and active (Fig. 1B), and a comparable trend was observed among 10 melanoma tissue samples (Fig. S2). To further extend this observation, we searched to establish a similar relationship in a larger number of melanoma tissue samples. Interestingly, examination of transcriptomic (RNA-seq) and methylomic (Infinium methylation assay) data from the Cancer Genome Atlas (TCGA), revealed a significant association between expression of CT-GABRA3 and hypermethylation of the downstream GABRA3 promoter in melanoma (Fig. 1C). Of note, this association was not affected by gender, thereby excluding a process related to X chromosome inactivation (supplementary Fig. S3).

Concurrent CT-GABRA3 activation and GABRA3 hypermethylation in lung adenocarcinoma. CT-GABRA3 is expressed not only in melanoma, but also in lung cancer. We decided to verify the association between hypomethylation/activation of CT-GABRA3 and hypermethylation of GABRA3 in this tumor type. To this end, we first exploited the Database of Transcription Start Sites (DBTSS), which contains various multi-omics data for a set of human lung adenocarcinoma (LUAD) cell lines ${ }^{22}$. Twenty three cell lines were grouped according to the status of expression of $C T$-GABRA3, which was defined on the basis of RNA-seq data $^{15}$. Among the 23 LUAD cell lines, 13 (57\%) scored positive for CT-GABRA3 (as well as MAGEA6) activation (Fig. 1D). Using Methyl-seq datasets, we then evaluated the DNA methylation levels of MAGEA6/CT-GABRA3 and GABRA3 promoters in the different LUAD cell lines. The results revealed that CT-GABRA3 activation in these cell lines is associated with hypomethylation of its promoter, as expected, but also with hypermethylation of the promoter region of GABRA3 (Fig. 1D). To verify if this association also pertains in vivo in lung adenocarcinoma tissue samples, we resorted to the analysis of TCGA datasets (Fig. 1E). This confirmed significant association between $C T$-GABRA3 activation and GABRA3 promoter hypermethylation in lung adenocarcinoma.

DNA hypermethylation extends all over the CT-GABRA3 transcription unit. The 530 kb genomic segment covered by the $C T$-GABRA3 transcript variant comprises another gene with brain-specific expression, $G A B R Q$ (Fig. 2A). Unlike GABRA3, GABRQ is normally transcribed in the opposite direction to CT-GABRA3. Examination of Methyl-seq data from the DBTSS database revealed that CpGs within the GABRQ promoter also displayed increased methylation in LUAD cell lines that express CT-GABRA3 (Fig. 2A). A similar observation was made for most other CpGs assessed within the $530 \mathrm{~kb}$ locus, thereby suggesting that CT-GABRA3 transcription exerts a regional effect of DNA hypermethylation (Fig. 2A). To evaluate the extent of this effect, changes of $\mathrm{CpG}$ methylation levels were analyzed in regions located immediately upstream (region A) and downstream (region C) of the locus defined by the CT-GABRA3 transcription unit, i.e. the entire region covered by CT-GABRA3 transcription (region B; Fig. 2B). Methyl-seq data in LUAD cell lines indicated that whereas CTGABRA3 activation was associated with DNA hypermethylation within its transcription unit (region B), it was instead associated with reduced DNA methylation levels in the neighboring regions A and C. This likely reflects 
A

CT-GABRA3



C

GABRA3 promoter methylation in melanoma tissues (TCGA) :

$\square C T-G A B R A 3-(\mathrm{n}=292)$

CT-GABRA3 + $(\mathrm{n}=177)$

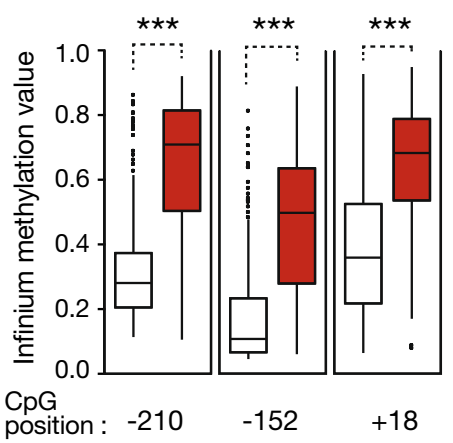

D



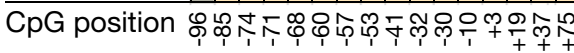
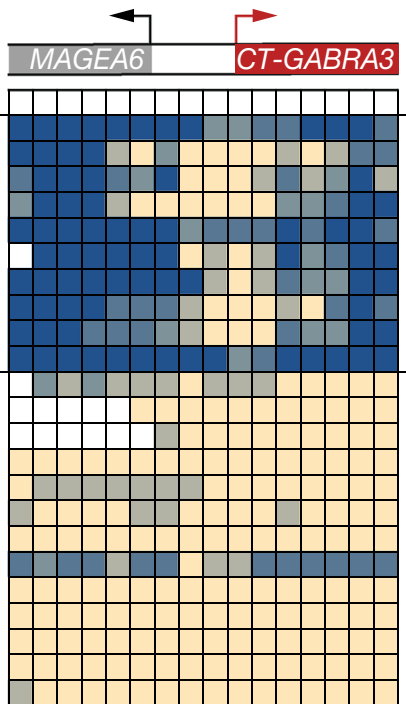

CpG methylation level
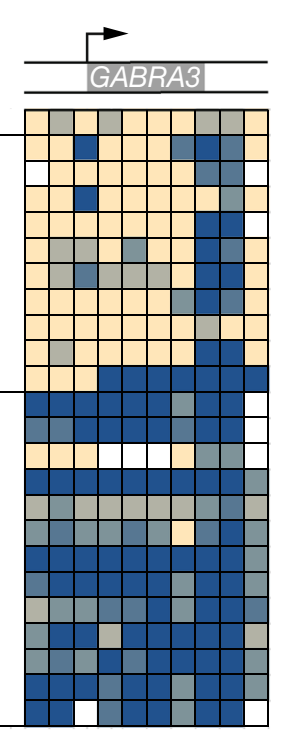



E

GABRA3 promoter methylation in lung adenocarcinoma tissues (TCGA) :

$\square$ CT-GABRA3 - $(\mathrm{n}=428)$
$\square C T-G A B R A 3+(\mathrm{n}=23)$

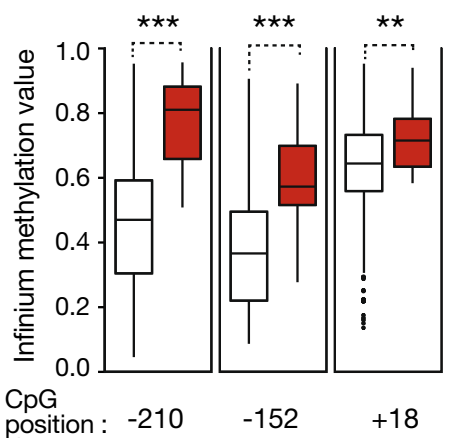

Figure 1. DNA hypomethylation of the MAGEA6/CT-GABRA3 promoter correlates with DNA hypermethylation of the GABRA3 promoter in melanoma and lung adenocarcinoma. (A) Schematic representation of the GABRA3 locus, with broken arrows indicating transcription start sites. The exon/intron structure of CT-GABRA3 and GABRA3 transcript variants is shown above. (B) Clonal bisulfite sequencing of MAGEA6/CT-GABRA3 and GABRA3 5'-regions. Vertical bars indicate location of CpG sites with positions relative to the transcription start site. Open and filled squares represent unmethylated and methylated CpG sites, respectively, and each row represents a single clone. CT-GABRA3 expression status (+) or (-) in melanoma cell lines is indicated. (C) Melanoma tissue samples from the TCGA were grouped according to CT-GABRA3 expression status (inferred from RNA-seq data), and the methylation level of three CPG sites embedded within the GABRA3 $5^{\prime}$-region (position relative to TSS) were determined through the analysis of Infinium methylation data (probe intensity ratio). ${ }^{* * *}$ Welch's t-test, adjusted $p$-value $<0.001$ (D) Methylation level of CpG sites within the MAGEA6/CT-GABRA3 and GABRA3 5'-regions in lung adenocarcinoma (LUAD) cell lines (CpG positions are expressed relative to the TSS). Methylation levels were calculated on the basis of Methyl-seq data from the DBTSS database. CT-GABRA3 expression status in LUAD cell lines was inferred from RNA-seq data. (E) The same analysis as described in $D$ was applied to lung adenocarcinoma samples from the TCGA. ${ }^{* *}$ and ${ }^{* *}$ Mann-Whitney test, adjusted $p$-value $<0.01$ and $<0.001$, respectively. 
A

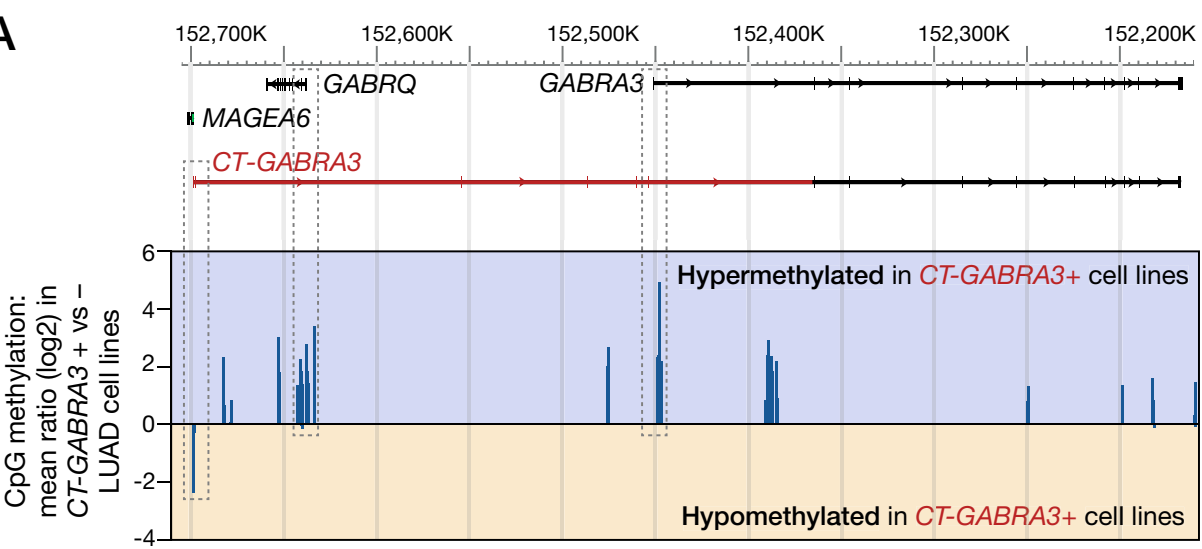

B
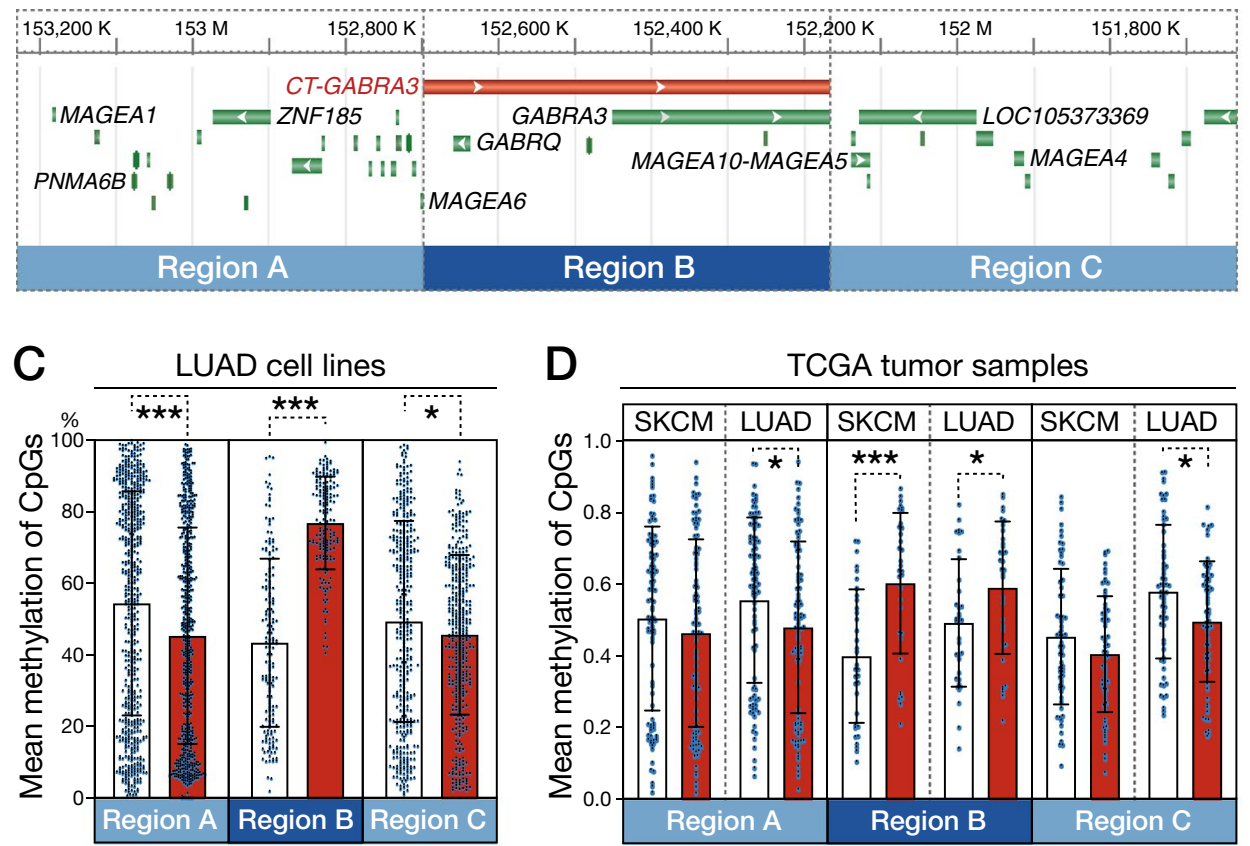

D

TCGA tumor samples

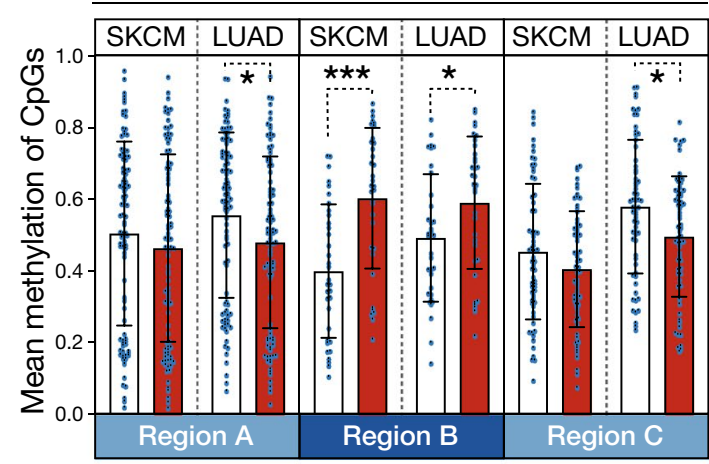

CT-GABRA3

CT-GABRA3 +

Figure 2. CT-GABRA3 expression correlates with hypermethylation of CpGs embedded within its entire transcription unit. (A) Using Methyl-seq data from DBTSS, CpG methylation ratios between CT-GABRA3positive and -negative LUAD cell lines were calculated. Values for all available CpGs within the GABRA3 locus are represented $(\log 2)$. Positive or negative values indicate hypermethylation or hypomethylation, respectively, of the CpG in CT-GABRA3-positive versus -negative LUAD cell lines. Hypermethylation includes CpGs within the promoter of GABRQ. (B) Schematic representation of the three defined $530 \mathrm{~kb}$ genomic segments, corresponding to the $C T$-GABRA3 transcription unit (Region B), and the two neighboring segments (Regions A and C). (C) LUAD cell lines were divided in two groups, according to CT-GABRA3 expression status, and Methyl-seq datasets from DBTSS were used to determine mean methylation levels (\% methylation) of all CpGs contained in each of the three genomic regions defined in (B). (D) A similar analysis, based on Infinium methylation data, was performed in melanoma (SKCM) and lung adenocarcinoma (LUAD) tissue samples from TCGA. ${ }^{*}$ and ${ }^{* * *}$ Welch's t-test, $p$-value $<0.05$ and $<0.001$, respectively.

the fact that activation of $C T$-GABRA3 occurs preferentially in tumor cells with global genome hypomethylation (Fig. 2C). Analysis of TCGA methylomic datasets confirmed the existence of a similar profile of regional DNA hypermethylation, limited to the $C T-G A B R A 3$ transcription unit, in melanoma and lung adenocarcinoma tissue samples (Fig. 2D).

Experimental evidence demonstrating that DNA hypomethylation/activation of CT-GABRA3 induces de novo methylation of overlapped CpGs. So far, our observations linking hypomethylation/activation of CT-GABRA3 and hypermethylation of CpG sites located within its $530 \mathrm{~kb}$ transcription unit 
were only correlative. To establish a direct link between these two events, we explored experimental results obtained by O'Neill and colleagues ${ }^{23}$, who generated three immortalized human fibroblast clones (of male origin) in which DNMT1 DNA methyltransferase was depleted following stable transfection of a specific shRNA vector (Fig. 3A). Interestingly, the authors reported that experimental depletion of DNMT1 resulted not only in losses, but also in gains of DNA methylation ${ }^{23}$. We decided to explore O'Neill's datasets to find out the changes that occurred within the MAGEA6/GABRA3 locus. Previous reports demonstrated that DNMT1 plays a key role in maintaining silencing of CG genes ${ }^{24-26}$. Consistently, cDNA microarray data revealed concurrent upregulation of MAGEA6 and (CT-)GABRA3 (microarray probes do not distinguish CT-GABRA3 and GABRA3 variants) in DNMT1-depleted cell clones (Fig. 3B). We then analyzed Infinium methylation assay datasets generated for the different groups of cells to evaluate methylation levels of CpGs located in either the CT-GABRA3 transcription unit (region B) or in the neighboring regions (region A and C, see Fig. 2B). The results revealed that, compared with the control cell line, all three DNMT1-depleted cell clones displayed significant increases of $\mathrm{CpG}$ methylation within the CT-GABRA3 transcription unit (region B, Fig. 3C). Methylation levels of CpGs located in adjacent regions $A$ and $C$, remained instead unchanged. Together these results demonstrate that hypomethylation/activation of CT-GABRA3 is linked with a process of de novo methylation of CpGs located within its transcription unit.

CT-GABRA3 transcription in LUAD cells correlates with regional increases in H3K36me3. We next searched to determine if CT-GABRA3 transcription also modifies histone marks within the overlapped genomic region. To this end, we analyzed ChIP-seq datasets of LUAD cell lines via the DBTSS platform, in order to evaluate the level of various histone marks around the transcription start site of GABRA3. These analyses revealed that CT-GABRA3 transcription in LUAD cells correlates with a decrease in H3K27me3 (Fig. 4A). This is consistent with initial presence of this mark, and its loss upon DNA hypermethylation through the previously described process of "epigenetic switch ", whereby promoter silencing shifts from a H3K27me3-mediated to a DNA methylation-mediated mechanism (supplementary Fig. S4, and ${ }^{27}$ ). Strikingly, an enrichment in H3K36me3 within the $5^{\prime}$-region of GABRA3 was also observed (Fig. 4A). We observed no significant change in the repressive histone mark $\mathrm{H} 3 \mathrm{~K} 9 \mathrm{me} 3$. Activating histone marks $\mathrm{H} 3 \mathrm{~K} 4 \mathrm{me} 3$ and $\mathrm{H} 3 \mathrm{ac}$ remained low in both $C T$ GABRA3-positive and -negative cell lines (Fig. 4A), consistent with the fact that GABRA3 is repressed in the two groups of cell lines. Similar observations were made for the GABRQ promoter, although the promoter showed a significant decrease of $\mathrm{H} 3 \mathrm{~K} 9 \mathrm{me} 3$ levels in CT-GABRA3-expressing cell lines (supplementary Fig. S5).

$\mathrm{H} 3 \mathrm{~K} 36 \mathrm{me} 3$ is classically enriched over the body of actively transcribed genes, as it is deposited along with the transcription machinery. It has been shown that $\mathrm{H} 3 \mathrm{~K} 36 \mathrm{me} 3$ favors local DNA methylation by attracting DNMT3 methyltransferases ${ }^{17,19,21}$. Inspection of the distribution of $\mathrm{H} 3 \mathrm{~K} 36 \mathrm{me} 3$ within the entire $530 \mathrm{~kb}$ genomic region covered by $C T$-GABRA3 transcription, revealed that enrichment of this histone mark in CT-GABRA3-positive LUAD cell lines already becomes apparent 15 to $20-\mathrm{kb}$ downstream of the transcription start site, and extends up into the GABRA3 promoter and beyond (Fig. 4B). Examination of ChIP-seq signals in neighboring segments (regions A and C, see Fig. 2B), indicated that increases in $\mathrm{H} 3 \mathrm{~K} 36 \mathrm{me} 3$ were limited to the region overlapped by $C T$-GABRA3 transcription (region B, Fig. $4 \mathrm{C}$ ). Together, these observations suggest that $C T$-GABRA3 transcription is accompanied by deposition of the H3K36me3 histone mark, and leads thereby to increased susceptibility of the entire transcription unit to DNA hypermethylation. This model explains how DNA hypomethylation, and concurrent transcriptional activation, can be connected with hypermethylation of adjacent promoters (Fig. 4D).

Other gene promoters displaying DNA hypermethylation in association with overlapping transcription in lung adenocarcinoma cells. An important issue was to determine if genes other than $G A B R A 3$ and $G A B R Q$, and in particular tumor suppressor genes, rely on a similar process of DNA hypomethylation-induced overlapping transcription to become hypermethylated in tumors. To this end, we examined the RNA-seq and Methyl-seq data obtained from LUAD cell lines (DBTSS) by applying a computational selection procedure to identify genomic loci that displayed the following features: i) ectopic activation in at least one LUAD cell line of a transcript that is not expressed in normal lung, ii) the ectopic transcript overlaps one or several downstream promoter(s) in either sense or anti-sense orientation, iii) the downstream overlapped promoter(s) (OPr) is(are) unmethylated in normal lung, and its hypermethylation is correlated with activation of the overlapping transcript (OTr) (Fig. 5A). This led to a list of 35 genomic loci, besides that containing GABRA3 and GABRQ. In three of these loci, activation of the overlapping transcript was correlated with DNA hypermethylation in not only one but two overlapped genes. Hence, our search identified 38 genes showing promoter hypermethylation in association with activation of an overlapping transcript in LUAD cell lines (Fig. 5B, supplementary Table S1). Overlapped promoters were located $2 \mathrm{~kb}$ to $128 \mathrm{~kb}$ downstream of the $\mathrm{OTr}$ transcription start site, in either sense or antisense orientation, and generally contained a high density of CpGs (Fig. 5B,C). Moreover, examination of ChIP-seq data revealed that $87 \%$ of the overlapped promoters displayed significant enrichment of H3K36me3 in the LUAD cell lines that express the corresponding overlapping transcript (Pearson correlation coefficient $>0.5$, adjusted $p$-value $<0.05$; Fig. $5 \mathrm{~B}$ ), thereby supporting the involvement of a silencing mechanism similar to that described for GABRQ and GABRA3 (Fig. 4D). Interestingly, eight among the overlapped hypermethylated genes (WT1, PAX6, GNAS, EPB41L1, CSMD1, CPEB1, RERG, and $S M A D 6$ ) were previously reported to exhibit tumor suppressive functions.

DNA demethylation accounts for the ectopic activation of several overlapping transcripts. We next searched to determine if DNA hypomethylation accounted for activation of the overlapping transcripts in the genomic loci we selected. To this end, we first exploited bisulfite-seq data from normal human tissues in order to sort out OTrs that have their promoter initially methylated in normal lung ( ${ }^{\mathrm{me}} \mathrm{CpG} \geq 50 \%$, Fig. $5 \mathrm{~B}$ ). 
A

$\underline{\text { O'Neill study }}$

DNMT1 depleted cell clones

pSh-DNMT1

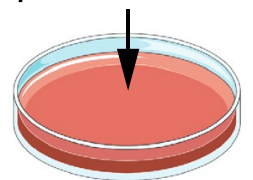

KD.D8

hTERT

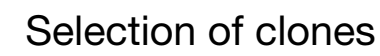

fibroblasts

B


C

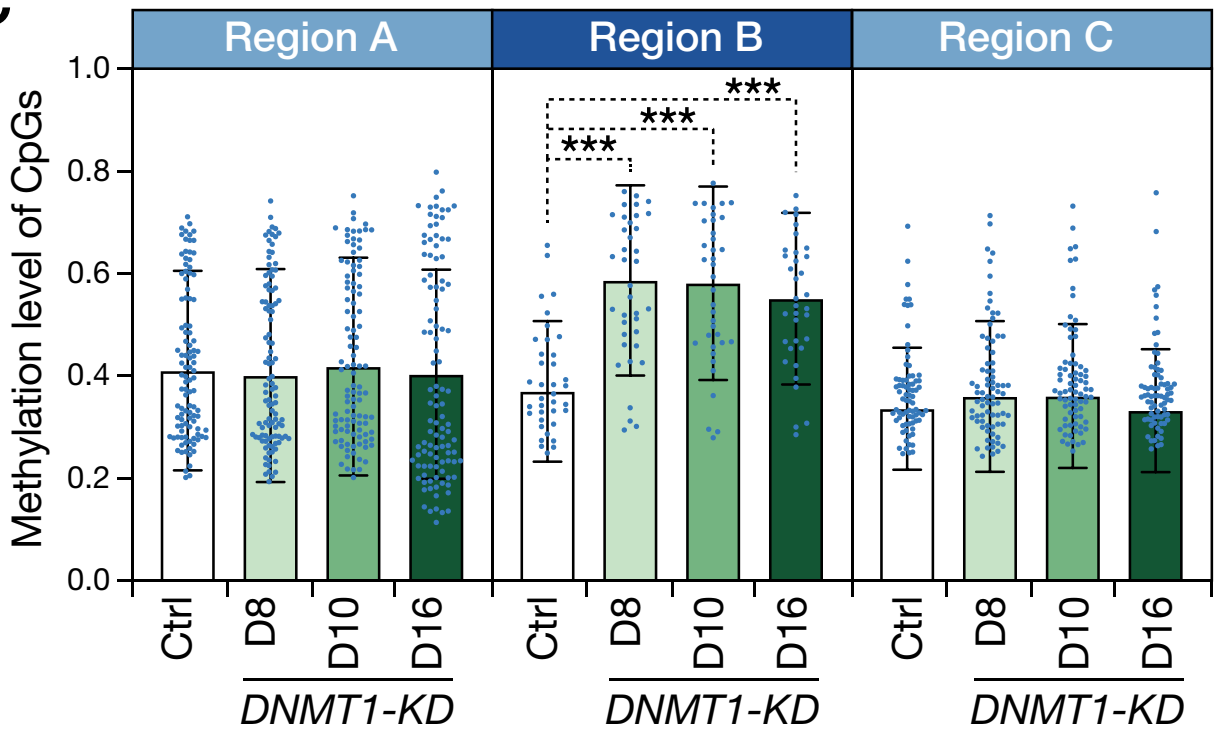

Figure 3. Experimental hypomethylation/activation of $C T$-GABRA3 induces de novo methylation of CpGs within its transcription unit. (A) Diagram depicting O'Neill's experiment. Clones were derived from hTERTimmortalized human male fibroblasts transfected with an anti-DNMT1 shRNA producing vector (pShDNMT1). (B) cDNA microarray data were analyzed to determine relative mRNA levels of DMNT1, MAGEA6 and (CT-)GABRA3 (the two transcript variants cannot be distinguished by microarray), in the control hTERT fibroblast cell line (Ctrl) and in the three DNMT1-depleted clones (D8, D10 and D18). (C) Infinium methylation data were used to determine mean methylation levels of all CpGs (mean probe intensity ratios) in the control cell line and the DNMT1-depleted clones. Analyses are provided for the CT-GABRA3 transcription unit (Region B), and the two neighboring segments (Regions A and C, see Fig. 2B). ${ }^{* *}$ One-way paired ANOVA test with Dunnett's correction, $p$-value $<0.001$. 
A Histone modifications within GABRA3 5'-region $(-1 \mathrm{~kb} /+1 \mathrm{~kb})$ in LUAD cell lines $\square$ CT-GABRA3 - $(\mathrm{n}=11) \quad \square$ CT-GABRA3 + $(\mathrm{n}=15)$

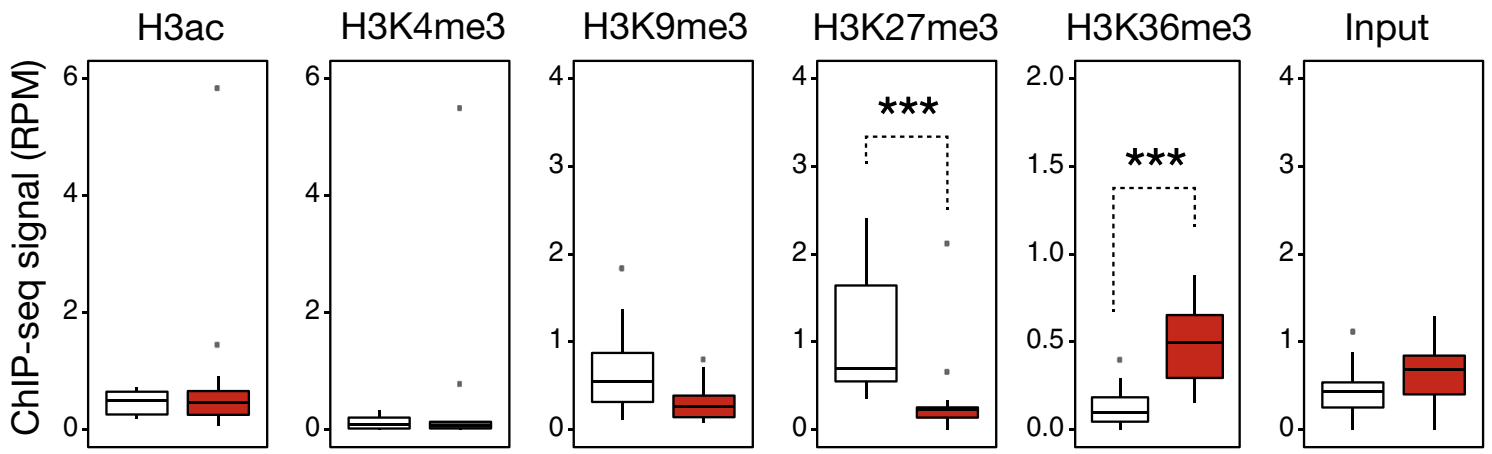

B



C
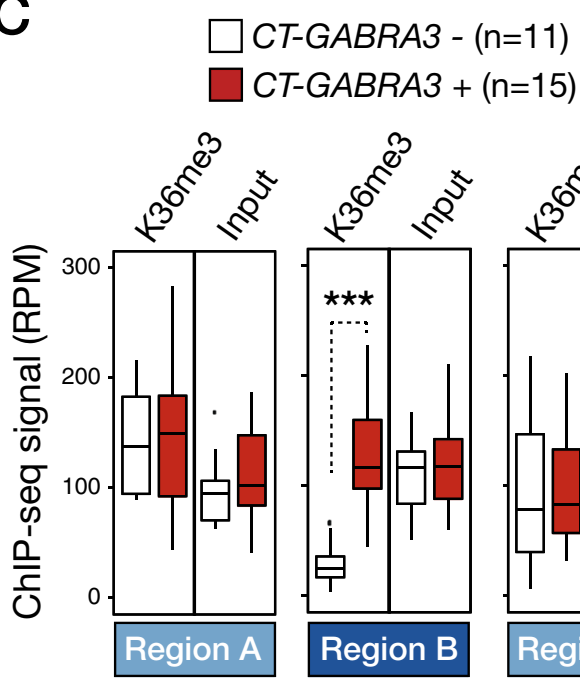
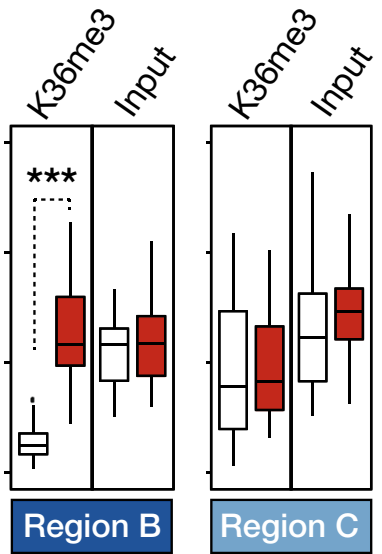

D

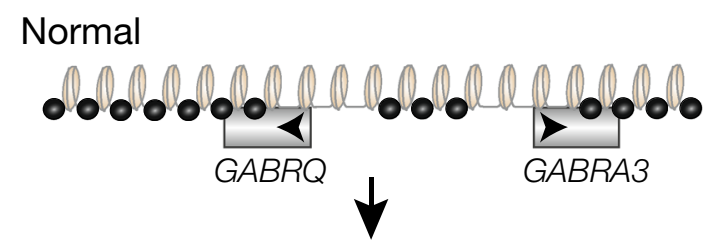

Tumor CT-GABRA3

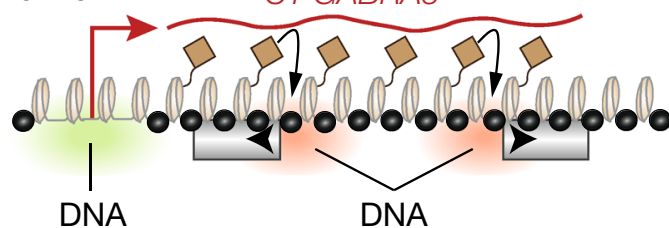

hypomethylation hypermethylation

Figure 4. CT-GABRA3 transcription correlates with regional increases in H3K36 trimethylation. (A) ChIP-seq results for the indicated histone modifications were extracted from the DBTSS platform, and mean amounts of reads corresponding to the GABRA3 $5^{\prime}$ region $(-1 \mathrm{~b} /+1 \mathrm{~kb})$ were compared in LUAD cell lines that do or do not express CT-GABRA3. ${ }^{\star * *}$ Mann-Whitney test, adjusted $p$-value $<0.001$. (B) ChIP-seq profile for H3K36me3 (DBTSS browser) within the entire CT-GABRA3 transcription unit, in 3 LUAD cell lines that do not express CT-GABRA3 (-) and 3 that do express it (+). (C) Mean amounts of ChIP-seq reads for H3K36me3 or control input in the three defined $530 \mathrm{~kb}$ genomic segments (Region B: CT-GABRA3 transcription unit; Regions A and C: neighboring segments), were compared in LUAD cell lines that do or do not express CT-GABRA3. ${ }^{* * *}$ Mann-Whitney test, $p$-value $<0.001$. (D) Model establishing the link between DNA hypomethylation/activation of CT-GABRA3 and DNA hypermethylation of $G A B R Q$ and $G A B R A 3$ promoters in tumor development. 
A
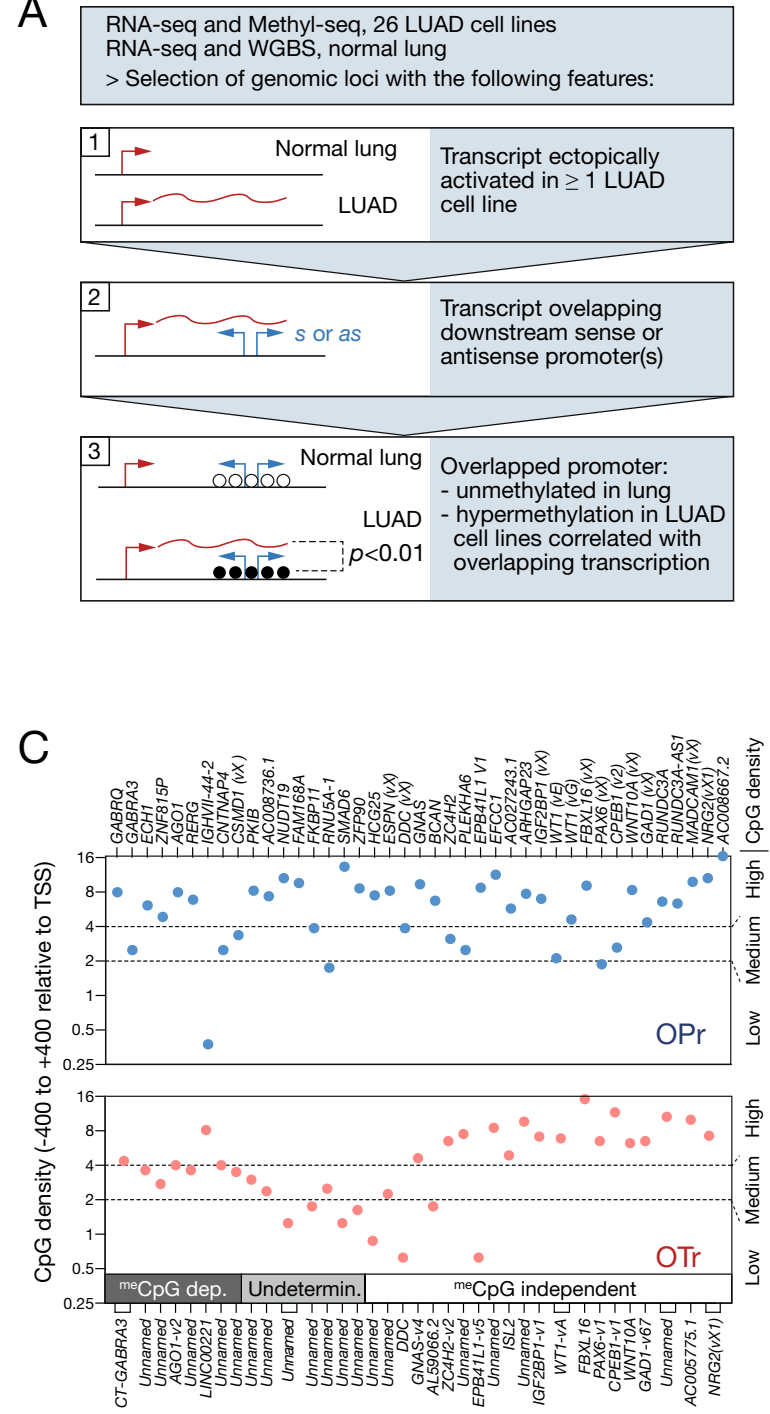

B

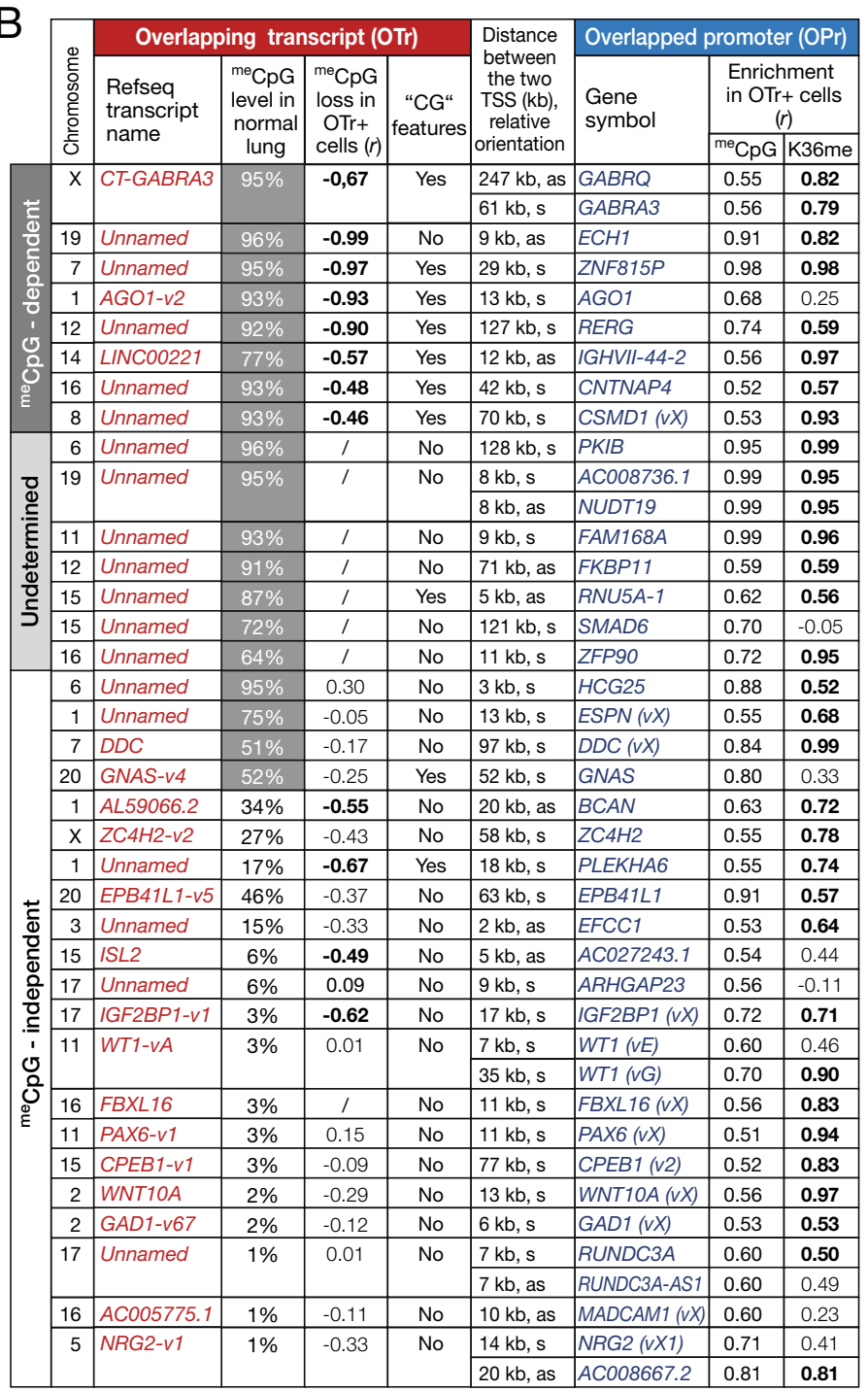

Figure 5. Identification of other genomic loci displaying correlation between DNA hypermethylation of an overlapped promoter (OPr) and activation of an overlapping transcript (OTr) in LUAD cell lines. (A) Flowchart of the bioinformatics selection procedure. Broken arrows indicate positions of transcription start sites, wavy lines correspond to overlapping transcription, and empty or filled circles represent unmethylated or methylated CpGs, respectively. (B) Features of selected genomic loci. For OPrs, the table provides: distance from TSS of OTr; gene symbol (with transcript variant according to NCBI RefSeq descriptions, or indicated as vX when the variant was undescribed); Pearson coefficient of correlation between OTr activation and promoter CpG methylation; Pearson coefficient of correlation between OTr activation and promoter H3K36me3 (coefficient in bold if adjusted $p$-value $<0.05$ ). For OTrs: name of transcript or transcript variant, or "unnamed" if not described in NCBI RefSeq; CpG methylation level of 5'-region in normal lung; Pearson coefficient of correlation between transcriptional activation and 5'-region CpG methylation level ("/" when methylation data were unavailable, coefficient in bold if adjusted $p$-value $<0.05$ ); cancer-germline ("CG") features, i.e. OTr is specifically expressed and its $5^{\prime}$-region demethylated in testis. OTrs were categorized according to their predicted dependency ( ${ }^{\mathrm{me}} \mathrm{CpG}$ dependent) or independency ( ${ }^{\mathrm{me}} \mathrm{CpG}$-independent) on DNA demethylation for transcriptional activation in LUAD cells (see text for criteria); several OTrs were categorized "Undetermined" because methylation data in LUAD cell lines were lacking. (C) The number of CpGs were calculated in the genomic segments located -400 to +400 relative to the TSS of OPrs and OTrs. Results are expressed as number of CpGs per $100 \mathrm{bp}$, and are plotted on a $\log 2$ scale. Sequences were categorized according to CpG density: (>4 CpG/100 bp: High, 2-4 CpG/100 bp: Medium, <2 CpG/100 bp: Low).

In addition, we examined Methyl-seq data from DBTSS, in order to identify OTrs that show significant association between activation and promoter demethylation among the 26 LUAD cell lines (Fig. 5B). Seven OTr genes (besides CT-GABRA3) fulfilled the two criteria, and were therefore considered as being DNA methyla- 
tion dependent. Importantly, 6 out of these 7 genes displayed typical "cancer-germline" features, i.e. preferential expression and promoter demethylation in testis (and for some in placenta), as well as sensitivity to induction upon treatment with the DNA demethylating agent $5^{\prime}$-aza-deoxycytidine (5-azadC) (Fig. 5B, supplementary Fig. S6 and S7). Moreover, 6 of these OTr genes contained an intermediate density of CpGs within their $5^{\prime}$ region (Fig. 5C), a recognized characteristic of DNA methylation-regulated gene promoters ${ }^{28}$. For 7 other OTr genes, high promoter methylation was observed in normal lung, but Methyl-seq data in LUAD cell lines were lacking. Dependency on DNA methylation could therefore not be determined for these genes. The remaining $17 \mathrm{OTr}$ genes were considered to be regulated by mechanisms not involving DNA methylation (Fig. 5B). Together, our selection procedure in LUAD cell lines led to the identification of 7 genes besides GABRQ and GABRA3 (ECH1, ZNF815P, AGO1, RERG, IGHVII-44-2, CNTNAP4, CSMD1) that become hypermethylated in lung tumor cells most likely through a process of DNA hypomethylation-induced overlapping transcription. For RERG, which initially displays elevated mRNA levels in normal lung (mean TPM $=16.38$, GTEx), we were able to show that its expression was significantly downregulated in the LUAD cell lines that express the overlapping transcript (supplementary Fig. S8). Moreover, we found that hypermethylation of many other genes are also associated with transcriptional overlap (Fig. 5B), but in these cases activation of the overlapping transcript did not appear to be due to promoter DNA demethylation.

DNA hypomethylation-induced transcriptional overlap is linked with promoter hypermethylation of PTPRO and RERG tumor suppressor genes. We chose to further investigate the RERG locus on chromosome 12, as it turned out that the OTr in this region overlaps not only one, but two genes with previously reported tumor suppressor functions: RERG (RAS Like Estrogen Regulated Growth Inhibitor), a negative regulator of the RAS/MAPK pathway and inhibitor of cell proliferation and tumor formation ${ }^{29,30}$; and PTPRO (Protein Tyrosine Phosphatase Receptor Type O), a phosphatase that counteracts the activity of tyrosine kinases, and modulates cell cycle progression and apoptosis ${ }^{31,32}$.

Examination of RNA-seq data with the Splice Junctions analysis tool of the Integrative Genome Viewer (IGV) confirmed the presence of a transcript overlapping PTPRO and RERG promoters in testis and several LUAD cell lines (Fig. 6A). The OTr was therefore named CT-RERG (Cancer-Testis RERG). RT-PCR experiments and RNA-seq data in healthy tissues revealed that CT-RERG is expressed not only in testis but also in placenta (Fig. 6B, and supplementary Fig. S6), a feature shared by several CG genes (Fig. 6B). Despite the presence of the entire $R E R G$ open reading frame in the CT-RERG mRNA, this transcript variant appeared as a poor substrate of RERG protein translation, probably due to the presence of short upstream open reading in the specific 5 ' exons (supplementary Fig. S9).

Analysis of bisulfite-seq datasets showed that CpG sites located around the TSS (-/+400 bp) of CT-RERG are highly methylated in normal somatic tissues and instead almost completely unmethylated in sperm (Fig. 6C). Evidence supporting a primary role of DNA methylation in the regulation of CT-RERG was provided by the observation that this transcript was induced upon 5-azadC treatment in all of three lung tumor cell lines, which were available in our lab and did not initially express the gene (Fig. 6D).

As for CT-GABRA3, we observed that transcriptional activation of CT-RERG in LUAD cell lines was accompanied by increases in H3K36 tri-methylation over the entire $240 \mathrm{~kb}$-long transcription unit, while neighboring regions remained unaffected (Fig. 6E,F). Consistently, whereas the CT-RERG transcription unit (region B) showed increased DNA methylation in expressing LUAD cell lines, neighboring regions A and C displayed instead reduced DNA methylation levels in these cell lines (Fig. 6G). Analyses of the RERG locus were further extended to in vivo tumor samples. Examination of TCGA datasets demonstrated that $C T$-RERG transcription is significantly correlated with PTPRO and RERG promoter hypermethylation in lung adenocarcinoma tissues (Fig. 6H). Since PTPRO has also been reported to exert a tumor suppressor function in hepatocellular carcinoma ${ }^{31}$, we analyzed corresponding TCGA datasets to verify association between CT-RERG expression and PTPRO hypermethylation in this tumor type. The results confirmed increased frequencies of PTPRO hypermethylation in the hepatocellular carcinoma samples that express CT-RERG (Fig. 6I). Together these observations confirm that DNA hypomethylation is associated with CT-RERG expression, and consequently with an increased propensity for PTPRO and $R E R G$ promoters to become hypermethylated. We noticed however, that a few CT-RERG-negative tumor samples nevertheless displayed DNA hypermethylation of PTPRO and RERG (Fig. 6H,I), thereby suggesting that transcriptional overlap may not be the only mechanism directing epigenetic silencing onto these promoters.

\section{Discussion}

It is currently proposed that DNA hypomethylation contributes to tumor progression by inducing genome instability, and by activating genes with oncogenic potential ${ }^{13,33}$. Our study now raises the interesting, and paradoxical, possibility that it also favors tumor development by contributing indirectly to the repression of tumor suppressor genes. We found indeed that focal DNA hypomethylation in tumor cells can lead to aberrant activation of transcripts that overlap downstream promoters and trigger their hypermethylation. Our work establishes therefore an unrecognized connection between DNA hypomethylation and DNA hypermethylation in tumors. This epigenetic coupling, however, applies to discrete genomic sites, and is therefore compatible with the accepted notion that genome-wide DNA hypomethylation is not associated at the global level with higher frequencies of DNA hypermethylation events ${ }^{5}$. Tumor-type specific patterns of DNA hypomethylation and hence of overlapping transcript activation, may instead be partly responsible for the selectivity of DNA hypermethylation events that is observed among tumors of different origins $\mathrm{s}^{34,35}$. For instance, hypermethylation of PTPRO and RERG promoters was only occasionally detected in renal carcinoma, a tumor type known to display infrequent hypomethylation and activation of CG genes ${ }^{12}$, and in which we found seldom activation of CT-RERG (supplementary Fig. S10). 
A

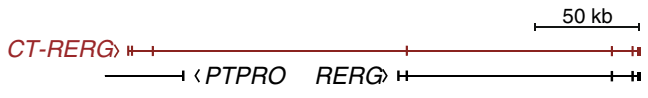

Normal tissues

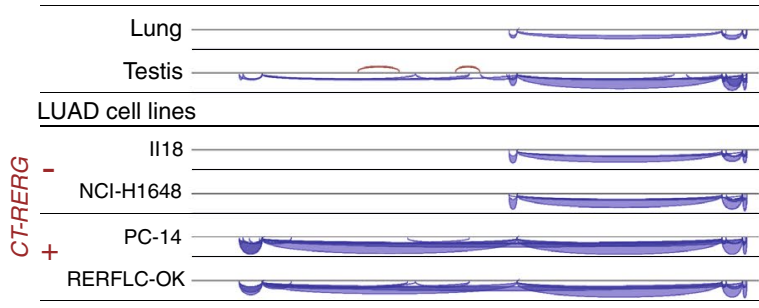

B

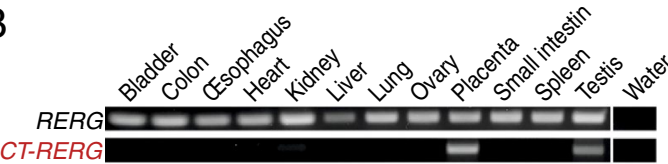

C
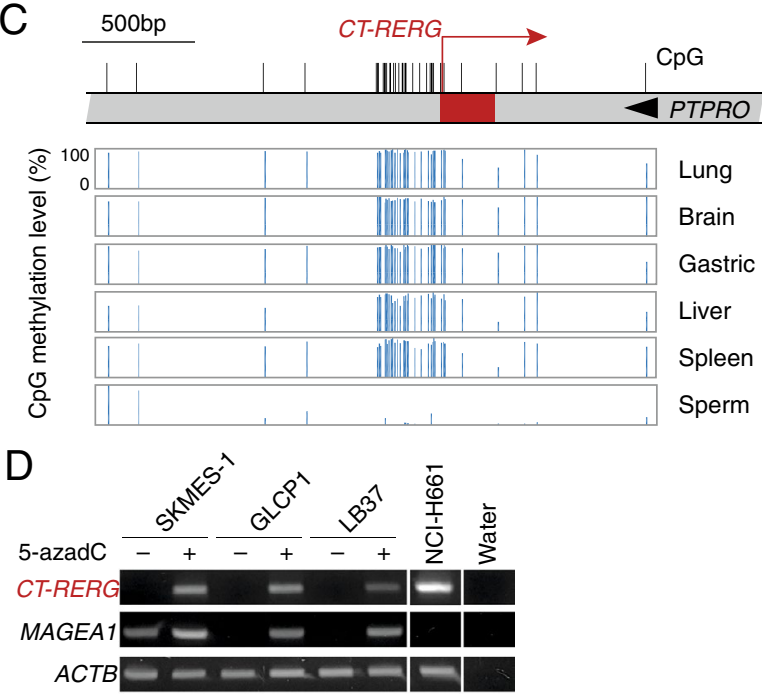

E



$\mathrm{F}$


$\mathrm{H}$

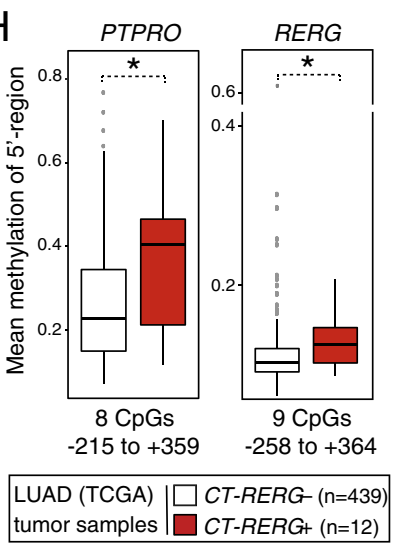

I

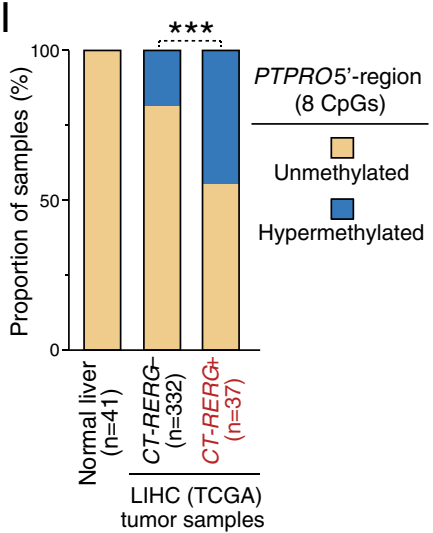

Figure 6. DNA hypomethylation-induced transcriptional overlap is associated with DNA hypermethylation of PTPRO and RERG tumor suppressor genes. (A) RNA-seq data from indicated samples were analyzed with IGV. Exon-intron structures are depicted on the top. (B) RT-PCR analyses in various normal tissues were performed with primers specific for either RERG or CT-RERG transcripts. (C) Bisulfite-seq data (NIH Roadmap epigenomics) revealing the methylation level (histograms) of CpG sites (vertical bars) surrounding the $C T-R E R G$ transcription start (broken arrow) in normal tissues. (D) $C T$-RERG-negative lung tumor cell lines were cultured with (-) or without (+) the DNA methylation inhibitor 5-azadC, and RT-PCR experiments were performed to test induction of CT-RERG mRNA. NCI-H661 lung tumor cell line was used as a positive control of CT-RERG expression. ACTB served as an internal control, and MAGEA1 as a control of 5-azadC induction. (E) Three $240 \mathrm{~kb}$ genomic regions were defined for subsequent analyses: CT-RERG transcription unit (B) and the two neighboring segments (A and $\mathbf{C}$ ). (F) Mean amounts of ChIP-seq reads for H3K36me3 or control input in genomic regions $\mathrm{A}, \mathrm{B}$ and $\mathrm{C}$ were compared in LUAD cell lines that do or do not express CT-RERG. ${ }^{*}$ MannWhitney test, $p$-value $<0.05$. (G) Mean methylation levels (\%, Methyl-seq data) of all CpGs contained in each of the three genomic regions were compared in CT-RERG-positive and -negative LUAD cell lines. ${ }^{* * *}$ MannWhitney test, $p$-value $\leq 0.0001$. (H) Mean methylation levels of all CpG sites embedded within the 5 'region of either the PTPRO ( $\mathrm{n}=8,-215$ to +359 relative to TSS) or RERG $(\mathrm{n}=9,-258$ to +364$)$ were compared in CT-RERG- and CT-RERG + LUAD tumor samples (TCGA). ${ }^{\star}$ Mann-Whitney test, $p$-value $<0.05$. (I) The proportion of samples with PTPRO hypermethylation was determined in TCGA samples of hepatocellular carcinoma (LIHC, $\mathrm{n}=369$ ) grouped according to the CT-RERG expression status. Considering that the mean $\mathrm{CpG}$ methylation values within the PTPRO 5'-region was $0.08(\mathrm{SD} \pm 0.014)$ in normal liver tissues $(\mathrm{n}=41)$, the region was considered hypermethylated in tumor samples where the mean $\mathrm{CpG}$ methylation value was $>0.2$. ${ }^{* * *}$ Fisher's exact test, $p$-value $<0.001$.

The role of overlapping transcription in directing de novo methylation of downstream promoters has been previously documented in normal developmental processes, notably during differentiation of embryonic stem cells, and for the establishment of parental imprinting marks in the germline $e^{17,20,36}$. It was proposed that, in 
tumors, the same process contributes to hypermethylation of alternative promoters that are embedded in the body of transcribed genes ${ }^{37}$. Our results support this concept, and provide a list of promoters that undergo hypermethylation in association with overlapping transcription in lung tumor cells. We show that overlapping transcripts sometimes initiate from a distant site, and are oriented in either sense or antisense direction compared with the hypermethylated promoter.

Although overlapping transcription is an efficient way of inducing de novo methylation of downstream CpGs, our analyses showed that overlapped promoters sometimes remained unmethylated. Lack of GABRQ/GABRA3 and $P T P R O / R E R G$ promoter hypermethylation was indeed observed in a fraction of tumor samples that nevertheless produced the overlapping transcripts. Hypermethylation of these promoters was also absent in testicular germ cells, where overlapping transcripts are expressed (supplementary Fig S11). It is therefore likely that, under certain conditions, promoters can resist overlapping transcription-induced DNA hypermethylation. Such a mechanism of resistance was previously reported in differentiating embryonic stem cells, and was correlated with elevated transcriptional activity of the overlapped promoter ${ }^{17}$. Moreover, we hypothesize that overlapped promoters are at higher risk of becoming hypermethylated in tumors that exhibit molecular imbalances favoring de novo DNA methylation, for instance through exacerbated activities of DNMT methyltranferases or impaired functioning of TET demethylases ${ }^{38-41}$.

Transcripts that overlap GABRA3 and RERG promoters are in sense orientation, and share all coding exons with the corresponding overlapped genes. Our analyses revealed, however, that these overlapping transcripts do not produce the corresponding proteins, possibly due to the presence of short upstream ORFs in the specific $5^{\prime}$ exons (supplementary Fig. S9) and ${ }^{16}$. When activated, these non-coding overlapping transcripts do have therefore the potential to cause loss of function of the overlapped gene. A corollary to this observation is that analyses of transcriptomic data in tumors might in some cases suggest that a gene is activated, when in fact activation pertains to a non-coding overlapping transcript that actually leads to loss of function of the gene. This may partly explain previous observations linking DNA hypermethylation with transcriptional activation ${ }^{42,43}$. Hence, high-resolution analyses of transcriptomic and methylomic data are required in order clearly understand the links between DNA methylation changes and gene expression in tumors ${ }^{44}$.

Most of the DNA hypomethylation-induced overlapping transcripts we identified correspond to previously unreferenced RNA species with unknown function. It is therefore unclear if they exert pro-tumoral functions. CT-GABRA3, however, was shown to produce two miRNAs (miR-105 and miR-767) with oncogenic potential $^{16,45}$, but the functional significance of the regulatory connection between CT-GABRA3 and GABRA3 remains unexplained.

It is hoped that a better understanding of the processes that underlie epigenetic alterations in tumors will lead to the development of novel tools for the diagnosis and therapy of cancer. In this line, establishing the pattern of expression of overlapping transcripts in tumor samples could serve to predict tumor suppressor genes that are at risk to become hypermethylated. Moreover, epigenetic anti-cancer therapies aiming at reactivating silenced tumor suppressor genes might benefit from the knowledge that some of these genes owe their hypermethylated status to a process of transcriptional overlap, and therefore to the specific contribution of druggable chromatin regulators, such as modifiers and readers of $\mathrm{H} 3 \mathrm{~K} 36 \mathrm{me} 3$ marks.

\section{Material and methods}

Cell culture. All human melanoma (MZ2-MEL, Mi13443, LB39-MEL, LB2667-MEL, Mi1811, SKMEL23) and lung cancer (SKMES-1, GLCP1, LB37, NCI-H661) cell lines were obtained from the Brussels branch of the Ludwig Institute for Cancer Research. Melanoma cell lines were cultured as previously described ${ }^{24}$. SKMES1, GLCP1 and LB37 cell lines were cultured in IMDM (Life Technologies) and NCI-H661 was cultured in RPMI (Life Technologies) medium, supplemented with $10 \%$ of fetal bovine serum (FBS, Sigma), $1 \times$ of non-essential amino acids (Life Technologies) and $1 \times$ penicillin/streptomycin (Life Technologies). HEK293T human embryonic kidney cells were purchased from Thermo Fisher and maintained in DMEM (Life Technologies), supplemented with GlutaMAX ${ }^{\mathrm{s}}$ (Life Technologies), $1 \times$ non-essential amino acids, $1 \times$ penicillin/streptavidin, and $10 \%$ FBS. Early passage human normal epidermal melanocytes were received from E. De Plaen (Ludwig Institute for Cancer Research, Belgium) and were cultured in Ham's F10 medium (Life Sciences) supplemented with $6 \mathrm{mM}$ Hepes, $1 \times$ MelanoMax supplement (Gentaur), and 10\% FBS.

5-azadC treatment. For 5-azadC induction experiments, tumor lines were grown to 60-70\% confluency, and then exposed to a single dose of $2 \mu \mathrm{M} 5$-aza-2'-deoxycytidine (Sigma-Aldrich) diluted in 1:1 acetic acid/ water. Treated cells were maintained in culture during 6 days before RNA extraction and RT-PCR analyses.

RT-PCR and qPCR analyses. RNA of tissue samples was purchased from Ambion (Life Technologies). RNA of cell lines was extracted using TriPure Isolation Reagent (Roche Diagnostics GmbH). Reverse transcription was performed on $2 \mu \mathrm{g}$ of total RNA using M-MLV Reverse transcriptase and random hexamers (Invitrogen). For PCR reactions, we used the DreamTaq Kit (Thermo Fisher Scientific), incorporating $1 / 40$ of the reverse transcription mixture in a final reaction volume of $20 \mu \mathrm{l}$. PCR reactions were visualized after electrophoresis in an ethidium bromide-stained agarose gel. For qPCR reactions, we used KAPA SYBR FAST (Sigma-Aldrich), incorporating $1 / 40$ of the reverse transcription mixture in a final reaction volume of $10 \mu \mathrm{l}$. All reactions were carried out according to the manufacturer's instructions. All primers are listed in the supplementary table S2.

Sodium bisulfite sequencing. Sodium bisulfite genomic sequencing of CT-GABRA3 and GABRA3 promoter regions was performed as previously described ${ }^{16}$. Primer used for nested PCR amplification of bisulfite treated DNA are listed in the supplementary table S2. 
Processing of public RNA-seq raw data. Fastq files of the 26 LUAD cell lines were downloaded from the DNA Data Bank of Japan (PRJDB2256). Fastq files of normal lung and testis tissues were downloaded from Sequence Read Archive of NCBI (PRJNA34535 \& PRJEB4337). All accession numbers are listed in supplementary table S3. Read alignment, de novo transcriptome assembly, and quantification of full-length referenced and unreferenced transcripts, were performed as described in the supplementary methods. For calculation of $C T$ GABRA3 and CT-RERG expression levels in LUAD cell lines and normal tissues, transcripts originating from the same TSS were summed. LUAD cell lines were considered positive for CT-GABRA3 or CT-RERG expression when the corresponding transcript showed a TPM $\geq 1$.

DNA methylation analyses in sperm, lung and LUAD cell lines. (1) Data collection: Whole genome bisulfite sequencing (WGBS) data for sperm and lung are provided by the NIH Roadmap epigenomics ${ }^{46}$. Normalized hg19 WGBS-seq data for sperm and lung were downloaded through the NCBI Gene Expression Omnibus, and were converted to hg38 using liftOver v1.10.0 R package. As corresponding data processing workflow does not allow multi-mapping of reads, methylation data for duplicated genomic regions, such as that containing the CT-GABRA3/MAGEA6 promoter ${ }^{15}$, were not available for sperm and lung. For LUAD cell lines, normalized hg38 target-captured bisulfite sequencing (Methyl-seq) data were downloaded from DBTSS v922. Only a fraction of genomic CpGs $(\sim 12 \%)$ are covered by the Methyl-seq method. Of note, three LUAD cell lines displaying ambiguous expression and DNA methylation results for the highly similar MAGEA6 and MAGEA3 genes ${ }^{15}$ were ignored for the analysis of the CT-GABRA3/MAGEA6 promoter methylation status. All accession numbers are listed in supplementary table S3. (2) Data analyses: For regional DNA hypermethylation analysis, we studied the methylation status of all CpGs located between $+1 \mathrm{~kb}$ of the TSS and up to the $5^{\prime}$ end of the OTr (= region B: $530 \mathrm{~kb}$ for $C T-G A B R A 3$ and $240 \mathrm{~kb}$ for $C T-R E R G$ ). Genomic segments of the same size were used to investigate $\mathrm{CpG}$ methylation levels in neighboring regions (regions $\mathrm{A}$ and $\mathrm{C}$ ). For the upstream region A, CpGs located within $1 \mathrm{~kb}$ upstream from the TSS of the OTr were excluded from the analysis. For analyses in LUAD cell lines, we only retained CpGs for which the methylation status could be determined in $>70 \%$ of the cell lines.

ChIP-seq data collection and analysis. Hg38 ChIP-seq data for H3ac, H3K4me3, H3K9me3, H3K27me3, H3K36me3 histone marks (and input) of the 26 LUAD cell lines were downloaded from DBTSS v9. To quantify histone modifications within promoter (TSS $+/-1 \mathrm{~kb}$ ) or genomic regions of interest in LUAD cell lines, we computed the total number of reads mapped to the corresponding genomic segment, divided by the sum of all reads generated in the same experiment, and multiplied by $10^{6}$ to obtain Reads Per Million (RPM) values.

TCGA consortium datasets. (1) Data collection: Normalized hg19 RNA-seq data with exon-level quantification and Infinium Human Methylation $450 \mathrm{~K}$ datasets for skin cutaneous melanoma (SKCM), lung adenocarcinoma (LUAD), liver hepatocellular carcinoma (LIHC), and kidney papillary cell carcinoma (KIRP) were downloaded from The Cancer Genome Atlas (TCGA) consortium ${ }^{47}$, using TCGAbiolinks v2.14.1 R-package ${ }^{48}$. $\mathrm{Hg} 19$ coordinates were converted to hg38 using liftOver v1.10.0 R package. Only unique primary (-01A) and metastatic (-06A) tumor samples, as well as unique normal adjacent tissues (-11A), for which both RNA-seq and Infinium methylation data were available, were analyzed. RNA-seq exon expression levels are expressed as Reads Per Kilobase per Million (RPKM). (2) Expression analyses: Since CT-GABRA3 and CT-RERG transcripts variants are not annotated in TCGA-derived datasets, we resorted to exon quantification to determine their expression status. Presence or absence of the canonical exon 1 allowed to distinguish CT-GABRA3 or CT-RERG transcript variants versus $G A B R A 3$ or $R E R G$ referenced transcripts, respectively. Thresholds were determined as follows: samples were considered positive for $C T$-GABRA3 expression if exon 1 displayed $\leq 0.1$ RPKM and exon $2 \geq 1$ RPKM; CT-RERG expression was positive when exon 1 displayed $\leq 0.4$ RPKM and exon $5 \geq 1$ RPKM. (3) DNA methylation analyses: For regional DNA hypermethylation analyses, Infinium methylation levels (beta values) were examined for all $\mathrm{CpG}$ probes located in regions $\mathrm{A}, \mathrm{B}$ and $\mathrm{C}$ regions, demarcated as described here above.

DNMT1 depletion experiments of O'Neill's study. Illumina HumanHT-12 V4.0 expression data (GSE90012) and Infinium Human Methylation $450 \mathrm{~K}$ data (GSE90011) were downloaded from NCBI Gene Expression Omnibus database. The following probes were used for expression analysis of the genes of interest: DNMT1 (ILMN_1715551), MAGEA6 (ILMN_2372681), and (CT-)GABRA3 (ILMN_1715551). For regional hypermethylation analyses, Infinium methylation levels (beta values) were examined for all CpG probes located in regions $\mathrm{A}, \mathrm{B}$ and $\mathrm{C}$ regions, demarcated as described here above.

Bioinformatics workflow for the identification of overlapped promoter hypermethylation. Identification of genomic loci that harbor an activated transcript leading to hypermethylation of a downstream promoter in LUAD cell lines, was performed by using a pipeline conducted in Perl programming language (scripts are available upon request), and applied to RNA-seq and Methyl-seq data of LUAD cell lines (DNA Data Bank of Japan, DBTSS), as well as RNA-seq and WGBS data of normal lung (Sequence Read Archive of NCBI, ENCODE). Initial processing of these RNA-seq datasets was described above. Details on the procedures to select transcripts activated in LUAD cell lines and potential overlapped promoters, and to establish correlations between overlapping transcript expression and overlapped promoter methylation, are described in the supplementary methods. 
Statistical analysis and graphical representations. Statistical analysis was computed in R v3.6.1 (http://www.R-project.org). Graphs and heatmaps were generated using R packages ggplot2 (v3.3.2) and ComplexHeatmap (v2.2.0). Benjamini-Hochberg correction was used for adjustment of $p$-values.

Received: 27 May 2021; Accepted: 17 August 2021

Published online: 30 August 2021

\section{References}

1. Flavahan, W. A., Gaskell, E. \& Bernstein, B. E. Epigenetic plasticity and the hallmarks of cancer. Science https://doi.org/10.1126/ science.aal2380 (2017)

2. Bird, A. DNA methylation patterns and epigenetic memory. Genes Dev. 16, 6-21 (2002).

3. Baylin, S. B. \& Jones, P. A. A decade of exploring the cancer epigenome-biological and translational implications. Nat. Rev. Cancer 11, 726-734. https://doi.org/10.1038/nrc3130 (2011).

4. Frigola, J. et al. Differential DNA hypermethylation and hypomethylation signatures in colorectal cancer. Hum. Mol. Genet. 14, 319-326 (2005).

5. Wilson, A. S., Power, B. E. \& Molloy, P. L. DNA hypomethylation and human diseases. Biochim. Biophys. Acta 1775, $138-162$. https://doi.org/10.1016/j.bbcan.2006.08.007 (2007).

6. Esteller, M. Epigenetics in cancer. N. Engl. J. Med. 358, 1148-1159 (2008).

7. Ehrlich, M. DNA hypomethylation in cancer cells. Epigenomics 1, 239-259 (2009).

8. Szyf, M., Pakneshan, P. \& Rabbani, S. A. DNA methylation and breast cancer. Biochem. Pharmacol. 68, 1187-1197 (2004).

9. De Smet, C. \& Loriot, A. DNA hypomethylation and activation of germline-specific genes in cancer. Adv. Exp. Med. Biol. 754, 149-166 (2013).

10. De Smet, C., Lurquin, C., Lethé, B., Martelange, V. \& Boon, T. DNA methylation is the primary silencing mechanism for a set of germ line- and tumor-specific genes with a CpG-rich promoter. Mol. Cell. Biol. 19, 7327-7335 (1999).

11. James, S. R., Link, P. A. \& Karpf, A. R. Epigenetic regulation of X-linked cancer/germline antigen genes by DNMT1 and DNMT3b. Oncogene 25, 6975-6985 (2006).

12. Wang, C. et al. Systematic identification of genes with a cancer-testis expression pattern in 19 cancer types. Nat. Commun. 7, 10499 . https://doi.org/10.1038/ncomms10499 (2016).

13. Van Tongelen, A., Loriot, A. \& De Smet, C. Oncogenic roles of DNA hypomethylation through the activation of cancer-germline genes. Cancer Lett. 396, 130-137. https://doi.org/10.1016/j.canlet.2017.03.029 (2017).

14. Whitehurst, A. W. Cause and consequence of cancer/testis antigen activation in cancer. Annu. Rev. Pharmacol. Toxicol. 54, 251-272. https://doi.org/10.1146/annurev-pharmtox-011112-140326 (2014).

15. Fain, J. S., Van Tongelen, A., Loriot, A. \& De Smet, C. Epigenetic coactivation of MAGEA6 and CT-GABRA3 defines orientation of a segmental duplication in the human X chromosome. Cytogenet. Genome Res. 159, 12-18. https://doi.org/10.1159/000502933 (2019).

16. Loriot, A. et al. A novel cancer-germline transcript carrying pro-metastatic miR-105 and TET-targeting miR-767 induced by DNA hypomethylation in tumors. Epigenetics 9, 1163-1171 (2014).

17. Jeziorska, D. M. et al. DNA methylation of intragenic CpG islands depends on their transcriptional activity during differentiation and disease. Proc. Natl. Acad. Sci. USA 114, E7526-E7535. https://doi.org/10.1073/pnas.1703087114 (2017).

18. Latos, P. A. et al. Airn transcriptional overlap, but not its lncRNA products, induces imprinted Igf2r silencing. Science 338, 1469-1472. https://doi.org/10.1126/science.1228110 (2012).

19. Baubec, T. et al. Genomic profiling of DNA methyltransferases reveals a role for DNMT3B in genic methylation. Nature 520, 243-247. https://doi.org/10.1038/nature14176 (2015).

20. Jones, P. A. Functions of DNA methylation: Islands, start sites, gene bodies and beyond. Nat. Rev. Genet. 13, 484-492. https://doi. org/10.1038/nrg3230 (2012).

21. Morselli, M. et al. In vivo targeting of de novo DNA methylation by histone modifications in yeast and mouse. Elife 4, e06205. https://doi.org/10.7554/eLife.06205 (2015).

22. Suzuki, A. et al. DBTSS/DBKERO for integrated analysis of transcriptional regulation. Nucleic Acids Res. 46, D229-D238. https:// doi.org/10.1093/nar/gkx1001 (2018).

23. O'Neill, K. M. et al. Depletion of DNMT1 in differentiated human cells highlights key classes of sensitive genes and an interplay with polycomb repression. Epigenetics Chromatin. 11, 12. https://doi.org/10.1186/s13072-018-0182-4 (2018).

24. Cannuyer, J., Loriot, A., Parvizi, G. K. \& De Smet, C. Epigenetic hierarchy within the MAGEA1 cancer-germline gene: Promoter DNA methylation dictates local histone modification. PLOS ONE 8, e58743 (2013).

25. Cannuyer, J., Van Tongelen, A., Loriot, A. \& De Smet, C. A gene expression signature identifying transient DNMT1 depletion as a causal factor of cancer-germline gene activation in melanoma. Clin. Epigenetics 7, 114. https://doi.org/10.1186/s13148-015-0147-4 (2015).

26. Loriot, A., De Plaen, E., Boon, T. \& De Smet, C. Transient down-regulation of DNMT1 methyltransferase leads to activation and stable hypomethylation of MAGE-A1 in melanoma cells. J. Biol. Chem. 281, 10118-10126 (2006).

27. Hinshelwood, R. A. et al. Aberrant de novo methylation of the p16INK4A CpG island is initiated post gene silencing in association with chromatin remodelling and mimics nucleosome positioning. Hum. Mol. Genet. 18, 3098-3109. https://doi.org/10.1093/hmg/ ddp251 (2009).

28. Weber, M. et al. Distribution, silencing potential and evolutionary impact of promoter DNA methylation in the human genome. Nat. Genet. 39, 457-466 (2007).

29. Finlin, B. S. et al. RERG is a novel ras-related, estrogen-regulated and growth-inhibitory gene in breast cancer. J. Biol. Chem. 276, 42259-42267. https://doi.org/10.1074/jbc.M105888200 (2001).

30. Zhao, W. et al. RERG suppresses cell proliferation, migration and angiogenesis through ERK/NF-kappaB signaling pathway in nasopharyngeal carcinoma. J. Exp. Clin. Cancer Res. 36, 88. https://doi.org/10.1186/s13046-017-0554-9 (2017).

31. Motiwala, T. et al. Suppression of the protein tyrosine phosphatase receptor type O gene (PTPRO) by methylation in hepatocellular carcinomas. Oncogene 22, 6319-6331. https://doi.org/10.1038/sj.onc.1206750 (2003).

32. Motiwala, T. et al. Protein tyrosine phosphatase receptor-type O (PTPRO) exhibits characteristics of a candidate tumor suppressor in human lung cancer. Proc. Natl. Acad. Sci. USA 101, 13844-13849. https://doi.org/10.1073/pnas.0405451101 (2004).

33. Gaudet, F. et al. Induction of tumors in mice by genomic hypomethylation. Science 300, 489-492 (2003).

34. Costello, J. F. et al. Aberrant CpG-island methylation has non-random and tumour-type-specific patterns. Nat. Genet. 24, 132-138 (2000).

35. Sproul, D. et al. Tissue of origin determines cancer-associated CpG island promoter hypermethylation patterns. Genome Biol. 13, R84. https://doi.org/10.1186/gb-2012-13-10-r84 (2012). 
36. Smallwood, S. A. \& Kelsey, G. D. novo DNA methylation: A germ cell perspective. Trends Genet. 28, 33-42. https://doi.org/10. 1016/j.tig.2011.09.004 (2012).

37. Pfeifer, G. P. Defining driver DNA methylation changes in human cancer. Int. J. Mol. Sci. https://doi.org/10.3390/ijms19041166 (2018).

38. Figueroa, M. E. et al. Leukemic IDH1 and IDH2 mutations result in a hypermethylation phenotype, disrupt TET2 function, and impair hematopoietic differentiation. Cancer Cell 18, 553-567. https://doi.org/10.1016/j.ccr.2010.11.015 (2010).

39. Ibrahim, A. E. et al. Sequential DNA methylation changes are associated with DNMT3B overexpression in colorectal neoplastic progression. Gut 60, 499-508. https://doi.org/10.1136/gut.2010.223602 (2011).

40. Noushmehr, H. et al. Identification of a CpG island methylator phenotype that defines a distinct subgroup of glioma. Cancer Cell 17, 510-522. https://doi.org/10.1016/j.ccr.2010.03.017 (2010).

41. O'Hagan, H. M. et al. Oxidative damage targets complexes containing DNA methyltransferases, SIRT1, and polycomb members to promoter CpG Islands. Cancer Cell 20, 606-619. https://doi.org/10.1016/j.ccr.2011.09.012 (2011).

42. Rauluseviciute, I., Drablos, F. \& Rye, M. B. DNA hypermethylation associated with upregulated gene expression in prostate cancer demonstrates the diversity of epigenetic regulation. BMC Med. Genomics 13, 6. https://doi.org/10.1186/s12920-020-0657-6 (2020).

43. Smith, J., Sen, S., Weeks, R. J., Eccles, M. R. \& Chatterjee, A. Promoter DNA hypermethylation and paradoxical gene activation. Trends Cancer 6, 392-406. https://doi.org/10.1016/j.trecan.2020.02.007 (2020).

44. Chen, Y. C. \& Elnitski, L. Aberrant DNA methylation defines isoform usage in cancer, with functional implications. PLoS Comput. Biol. 15, e1007095. https://doi.org/10.1371/journal.pcbi.1007095 (2019).

45. Zhou, W. et al. Cancer-secreted miR-105 destroys vascular endothelial barriers to promote metastasis. Cancer Cell 25, 501-515. https://doi.org/10.1016/j.ccr.2014.03.007 (2014).

46. Roadmap Epigenomics, C. et al. Integrative analysis of 111 reference human epigenomes. Nature 518, 317-330. https://doi.org/ 10.1038/nature14248 (2015).

47. Grossman, R. L. et al. Toward a shared vision for cancer genomic data. N. Engl. J. Med. 375, 1109-1112. https://doi.org/10.1056/ NEJMp1607591 (2016).

48. Colaprico, A. et al. TCGAbiolinks: An R/Bioconductor package for integrative analysis of TCGA data. Nucleic Acids Res. 44, e71. https://doi.org/10.1093/nar/gkv1507 (2016).

\section{Acknowledgements}

This work was supported by grants from the D.G. Higher Education and Scientific Research of the French Community of Belgium (Action de Recherches Concertées) and from the Fonds special de recherche (FSR) of the UCLouvain, Belgium. AL was supported by the de Duve Institute, Brussels, Belgium. AVT was a recipient of a Télévie grant from the FRS-FNRS, Belgium [\#7.4581.13]. AD received a fellowship from FRS-FNRS-FRIA, Belgium [\#1.E008.19]. Funding for open access charge: de Duve Institute, Brussels, Belgium.

\section{Author contributions}

J.S.F., A.L. and C.D.S. conceived and designed the experiments: J.S.F., A.D., A.V.T., and A.L. performed the experiments. J.S.F., A.L., A.D. and C.D.S. analysed the data. J.F.S. and C.D.S. wrote the paper.

\section{Competing interests}

The authors declare no competing interests.

\section{Additional information}

Supplementary Information The online version contains supplementary material available at https://doi.org/ 10.1038/s41598-021-96844-0.

Correspondence and requests for materials should be addressed to C.S.

Reprints and permissions information is available at www.nature.com/reprints.

Publisher's note Springer Nature remains neutral with regard to jurisdictional claims in published maps and institutional affiliations.

(c) (i) Open Access This article is licensed under a Creative Commons Attribution 4.0 International License, which permits use, sharing, adaptation, distribution and reproduction in any medium or format, as long as you give appropriate credit to the original author(s) and the source, provide a link to the Creative Commons licence, and indicate if changes were made. The images or other third party material in this article are included in the article's Creative Commons licence, unless indicated otherwise in a credit line to the material. If material is not included in the article's Creative Commons licence and your intended use is not permitted by statutory regulation or exceeds the permitted use, you will need to obtain permission directly from the copyright holder. To view a copy of this licence, visit http://creativecommons.org/licenses/by/4.0/.

(C) The Author(s) 2021 